\title{
Precise Forecast and Application of Time Delay Receiving Schedule for a New Generation of Polar Orbit Meteorological Satellite
}

\author{
Zhaohui Cheng, Manyun Lin, Cunqun Fan* \\ National Satellite Meteorological Center, Beijing, China \\ Email: ^fancq@cma.gov.cn
}

How to cite this paper: Cheng, Z.H., Lin, M.Y. and Fan, C.Q. (2018) Precise Forecast and Application of Time Delay Receiving Schedule for a New Generation of Polar Orbit Meteorological Satellite. Journal of Geographic Information System, 10, 142-149. https://doi.org/10.4236/jgis.2018.101006

Received: January 16, 2018

Accepted: February 9, 2018

Published: February 12, 2018

Copyright (c) 2018 by authors and Scientific Research Publishing Inc. This work is licensed under the Creative Commons Attribution International License (CC BY 4.0).

http://creativecommons.org/licenses/by/4.0/

\begin{abstract}
In order to finely predict the receiving schedule of the new generation of polar orbit meteorological satellite time-delay data and solve the problem of rapid positioning of lost data, this paper studies and proposes the satellite data recording and satellite program-controlled program, and designs the delay data receiving timeline precision forecasting method. It is concluded that the detection load of polar orbit meteorological satellite in our country has developed from single load to multiple loads, and the detection data need to be downloaded to the ground for processing and application. And as the satellite load increases and the accuracy of each payload detection and channel increases, the amount of probing data will further increase, which in turn will require further increase of the speed of data transmission in the earth. Due to the limitation of the space data transmission frequency band, under the prior art system, the increase of the satellite data transmission rate is limited. On the basis of understanding the working principle of Fengyun-3, the new transmission system will be implemented in terms of data source compression, channel coding, modulation and polarization multiplexing by exploring new weather transmission systems for meteorological satellites in the future upgrade and at the same time analyze ways to avoid inter-satellite interference in order to solve the contradiction between the increase of data volume and the resource of terrestrial data transmission in the existing system.
\end{abstract}

\section{Keywords}

Delay Data, Fine Prediction, Data Location

\section{Introduction}

Fengyun-3 meteorological satellite is China's second-generation polar orbiting 
meteorological satellite, the amount of delay data far exceeds the amount of realtime data, delayed data reception on the integrity of the global data has a great impact [1]. The first generation of China's polar weather satellite delay data reception by the three ground receiving stations located in Beijing, Guangzhou and Xinjiang in our country. There are seven transit orbits, but often there is a track to receive no delay data (star on the memory is empty), so we can only receive 6 track delay data, and in this case, the ground system can not accurately predict, only to be informed when the actual reception. China's second-generation polar orbit weather satellite delay data reception in China by Beijing, Guangzhou, Xinjiang, Jiamusi and Sweden Kiruna, five ground receiving stations to receive about 14 transit tracks per day, orbit relay will still be appearing on-board. Memory is empty; the ground [2] station will appear to receive delay data. The traditional prediction of receiving polar orbit meteorological satellite time-delay data is based on the time of satellite transit and the program of satellite program control, which does not consider the actual data storage on the satellite and the code rate of satellite decentralization that may result in the possibility of forecasting satellite transit reception time. There will be no delay data situation; ground receiving station delay data reception cannot be accurately predicted.

The meteorological satellites observe the surface of the earth and the atmosphere from outer space [3] [4]. They are characterized by high observational areas, wide observation areas and high frequency of observation [5]. It is a characteristic of large-scale dynamic observation of the ground [6]. A polar orbiting meteorological satellite can obtain global meteorological [7] data twice a day, and a stationary meteorological satellite can obtain weather images of nearly one quarter of the earth every 30 minutes [8]. Meteorological satellites not only can get a wide range of images of the Earth's surface and the cloud top [9], but also obtain quantitative meteorological data of the three-dimensional space atmosphere such as temperature, humidity [10], pressure and radiation, which plays an important role in weather forecasting and climate prediction [11]. Now the application of meteorological satellites has far exceeded the traditional meaning of the meteorological category [12], in the dynamic monitoring of ecological environment and natural disasters, as well as marine, agriculture, fisheries, aviation, navigation and so has a wide range of uses [13]. Fengyun-3 ground application system is a new design and construction of the system, which carries 11 instruments, of which 9 are for the first time on the Star [14]. The instrument's detection band covers UV, visible, infrared and microwaves [15]. The most prominent feature of this instrument is its ability to detect all-weather atmospheric conditions [16]. It provides initial field data for numerical weather forecasting and visible and infrared data for environmental monitoring up to $250 \mathrm{~m}$ resolution. Fengyun-3 detection data of large amount of data [17], transmission channels and requiring high time-consuming access, it is the earth's comprehensive remote sensing detection of large polar [18] orbiting satellites, in a space 
platform, using a variety of detection methods, simultaneous detection, can compare Good to meet the needs of meteorological observation.

The traditional receiving schedule of polar orbit meteorological satellite timedelay data only predicts the transit time of the satellite under the specified antenna elevation angle, and cannot predict the downlink data situation. With the development and upgrading of satellite technology, the technical level of terrestrial business systems is also getting higher and higher, and the requirements for forecasting delay data of polar orbit meteorological satellites are also getting higher and higher, and the delay data for each track is accurately predicted The amount of time delay data transmitted by satellites during transit and the observation time period of delay data received by each orbit, the time delay data source for timely discovering the integrity of postponed data reception and the after-analysis of poor quality at the ground receiving station very necessary.

\section{Realization of Fine Forecasting of Time Delay Data Receiving Schedule}

Accurately forecast time delay data reception schedule is based on the original satellite transit time table to increase the number of Fengyun-3 delay data reception time period, and the received data observation period.

Precisely forecasting the delay time of receiving data on the ground station, we must always know the state of the data in the satellite memory, the storage of delay data on the satellite during the transit, and the code rate of the satellite downlink.

Accurately forecast the ground station receives the load data observation period, we must master the program of satellite programs to record each transit, departure, the delay of data transmission, input into the memory of the load data period.

\subsection{Research on Orbit Prediction Method}

Analyze and study the advantages and disadvantages of many kinds of orbit prediction algorithms and select the prediction algorithm that is adaptable and matches with the orbit calculation of polar orbit meteorological satellite in China.

\subsection{Ground Receiving Range Forecasting Research}

According to the configurable station and antenna receiving range, the corresponding azimuth and time when the antenna passes through different station areas, the antenna receiving at different elevation angles and the length of satellite transit time at each receiving elevation angle are predicted.

\subsection{On-Time Delay Data Recording of the Forecast}

According to the program of satellite program, the on/off status of the satellite remote sensing equipment is extracted from the satellite telemetry data, and the delay data storage quantity is accurately calculated and predicted according to 
the code rate of each instrument recording data and the recorded time length.

The amount of data stored in each track during entry into the country

$=\Sigma$ Load data amount

$=\Sigma\left(\right.$ Load data record code rate ${ }^{\star}$ Recording time $)$.

It can be seen from Table 1 that the recording rate of the mid-resolution spectral imager and the scanning radiometer is different during day and night, and the time corresponding to the code rate needs to be strictly in accordance with the daytime and nighttime status of the satellite.

\subsection{Delay Data Ground Reception Time Forecast Study}

Delay time, the ground reception time is the data transmission time of satellite transit. Combined with the format of digital transmission and the proportion of delay data in the satellite decentralized data, the effective time period of the data when the satellite passes the delay of different stations is predicted.

Calculate the state of the recorded records in a day, divided into three states: MERSI and other instruments co-recorded state; other instruments record MERSI does not record the state; record stop state, record the state of the recordings stored every second and multiply the recording rate of the corresponding instrument to calculate the amount of solidified recording.

Number of stored records $=$ Recording status stored every second ${ }^{\star}$ Recording rate of corresponding instrument ${ }^{*}$ Constant factor.

Table 1. Delay link data corresponding to the instrument.

\begin{tabular}{|c|c|c|c|c|}
\hline Remote sensing equipment & Record code rate & MPT & DPT & Record storage \\
\hline Medium resolution spectral imager & $16 \mathrm{Mbps}$ & Medium resolution imaging spectrometer & & $\begin{array}{l}\text { Star point data } \\
\text { during the day }\end{array}$ \\
\hline Scanning radiometer (daytime) & $1.3308 \mathrm{Mbp} \sqrt{ }$ & Scanning radiometer & s & Global record \\
\hline Scanning radiometer (night) & $0.39924 \mathrm{Mbps}$ & & & \\
\hline Microwave imager & $100 \mathrm{Kbps}$ & & & Global record \\
\hline Infrared spectrometer & 4 packs $/ 6.4 \mathrm{~s}$ & & & Global record \\
\hline Ozone vertical detector & 1 package/64 s & Microwave imager & & Global record \\
\hline Total ozone detector & 1 package/8.16 s & Satellite engineering telemetry parameters & & Global record \\
\hline Earth radiation detector & 1 package $/ 4 \mathrm{~s}$ & Infrared spectrometer & & Global record \\
\hline Solar radiation monitor & $\begin{array}{c}1 \text { package } / 15 \mathrm{~s} \\
\text { (4 to } 16 \text { packets/track) }\end{array}$ & Earth radiation revenue detector & & Global record \\
\hline Microwave thermometer & 4 packs/2.667 s & Microwave thermometer & & Global record \\
\hline Microwave hygrometer & 4 packs/2.667 s & Total ozone detector & & Global record \\
\hline Space Environment Monitor & $1 \mathrm{pack} / 42 \mathrm{~s}$ & Ozone vertical detector & & Global record \\
\hline GNOS occultation detector & $200 \mathrm{Kbps}$ & & $\begin{array}{c}10 \text { packs } / 120 \mathrm{~s} \\
\text { (once a day) }\end{array}$ & Global record \\
\hline $\begin{array}{l}\text { Satellite engineering telemetry } \\
\text { data (GPS signal included) }\end{array}$ & 2 packs/s & Solar radiation monitor & & Global record \\
\hline
\end{tabular}




\subsection{Delay Data Time Forecast Software Research}

The forecast software is researched and developed by the National Satellite Meteorological Forecasting Center. The software is used to analyze the relationship between the data received on the ground and the data stored on the satellite and to determine the recording time of the data segment.

The amount of data stored in storage for a period of time $=$ the amount of data recorded in storage during exit - the amount of delayed data delivered during entry.

DPT reception time per track ${ }^{\star}$ DPT data transfer rate $=$ amount of data that can be dropped by this track.

Determine whether the amount of data that can be dropped by the track is greater than the number of stored records. If it is smaller than the number, it indicates that the track cannot be completely deallocated and the remaining data will be accumulated to the next track.

According to the state of the recordings stored every second, judge the time period for recording the MERSI and other instruments on a track.

\section{Application of Precise Prediction Algorithm}

According to the accurate prediction of the data receiving schedule, it is learned in advance when and where the real-time observed data of various loads on the satellite will be downloaded to which ground receiving station. So the ground system will load the global observation data by arc projected onto the earth (Figure 1), you can right click the properties of each arc, including the arc of the receiving time, the received track number, the received ground station and other information. When there is missing data in the puzzle, you can quickly pass the adjacent arc at the missing data to determine the missing attribute of the arc, so as to know the source of the missing data and locate the cause of the problem.

\section{Conclusion and Discussions}

Based on the analysis of the working principle and function of Fengyun-3

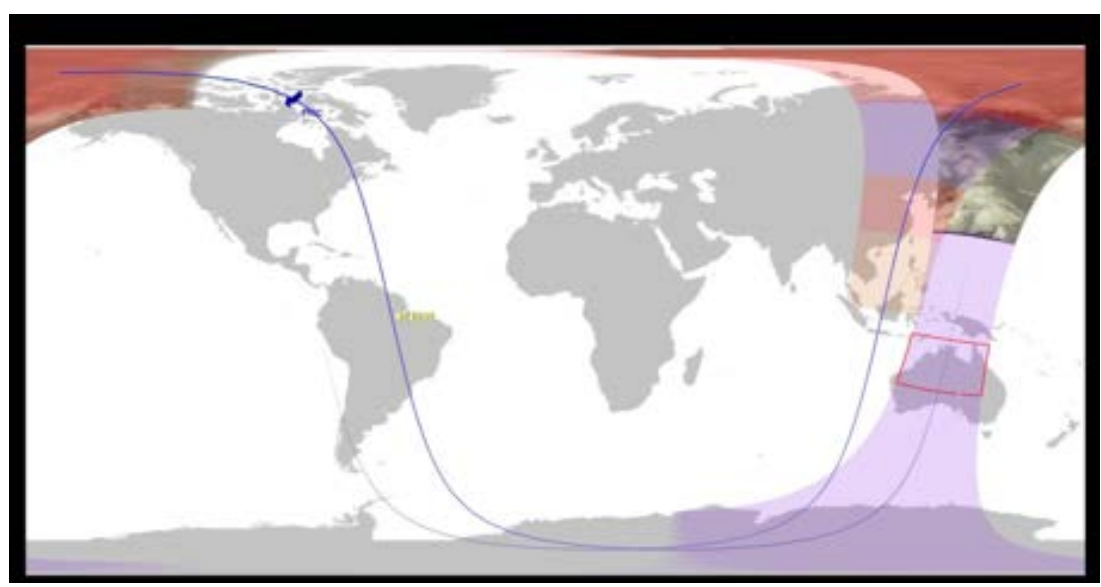

Figure 1. Global observational data projection. 
meteorological satellite, this paper presents satellite data recording and satellite program control, and designs a fine forecasting method of time delay data receiving schedule, so as to finely predict the receiving schedule of delay data of a new generation of polar orbit meteorological satellites and solve the problem of rapidly locating lost data.

1) The polar orbit meteorological satellite [19] in our country has been developed from Fengyun-1 to Fengyun-3 at present. The detection load of a star develops from a single load to multiple loads. The detection data need to be downloaded to the ground for processing and application. Star data transfer code rate from F1. 3308 Mbps to 300 Mbps. Subsequent satellites as the satellite load increase and the accuracy of the payload detection and channel increases [20], the amount of probing data will further increase, thus requiring further increase in the rate of data transfers to the earth. Due to the limitation of the data transmission space of the land space, under the prior art system, the increase of the satellite data transmission rate is limited [21].

2) Fengyun-3 satellite data transmission system uses a multi-carrier, unipolar, QPSK modulation, encoding using RS + (7.3/4) convolutional encoding. DPT channel occupies a bandwidth of $300 \mathrm{Mhz}$; the frequencies used by the ITU for air-to-ground [22] data transmission are located in the L band, the $\mathrm{X}$ band and the KA band, respectively, of which the L band is only $12 \mathrm{MHz}$ in bandwidth and is mainly used for real-time data, the X-band includes $7750 \mathrm{MHz}-7900$ MHZ and $8025 \mathrm{MHZ}-8400 \mathrm{MHZ}$. The former segment is mainly used for the transmission of medium-capacity real-time data due to its narrow bandwidth. The latter segment is widely used in the global data transmission at home and abroad. KA segment due to greater rain attenuation, and ground tracking technology are not mature; there is no application in orbit. That is, the use of X-band space for data transmission resources is limited.

3) In order to solve the contradiction between the increase of satellite data, transmission data volume and spatial resources in the existing system [23], it is necessary to explore the new meteorological satellite in the future to adopt a new transmission system. The new transmission system will compress the data source and encode the channel, modulation and polarization multiplexing and other aspects of the upgrade, at the same time, needs to analyze ways to avoid interstellar interference.

\section{Acknowledgements}

The work presented in this study is supported by National High-Tech R \& D Program (2011AA12A104).

\section{References}

[1] Lin, M., Zhao, X., Fan, C., Xie, L. and Wei, L. (2017) Study on Massive Vegetation Data Processing of FY-3 Based on RAM (h). Journal of Geoscience and Environment Protection, 5, 75-83. https://doi.org/10.4236/gep.2017.54007

[2] Lin, M., Zhao, X., Zi, X., Guo, P. and Fan, C. (2017) Classification of Meteorological 
Satellite Ground System Applications. Atmospheric and Climate Sciences, 7, 374381. https://doi.org/10.4236/acs.2017.73028

[3] Peterson, P.K., Simpson, W.R., Pratt, K.A., Shepson, P.B., Frieß, U., Zielcke, J., et al. (2015) Dependence of the Vertical Distribution of Bromine Monoxide in the Lower Troposphere on Meteorological Factors Such as Wind Speed and Stability. Atmospheric Chemistry \& Physics, 15, 1-19. https://doi.org/10.5194/acp-15-2119-2015

[4] Krauss, S., Fichtinger, B., Lammer, H., Hausleitner, W., Kulikov, Y.N., Ribas, I., et al. (2012) Solar Flares as Proxy for the Young Sun: Satellite Observed Thermosphere Response to an x17.2 Flare of Earth's Upper Atmosphere. Annales Geophysicae, 30, 1129-1141. https://doi.org/10.5194/angeo-30-1129-2012

[5] Hertig, E., Paxian, A., Vogt, G., Seubert, S., Paeth, H. and Jacobeit, J. (2012) Statistical and Dynamical Downscaling Assessments of Precipitation Extremes in the Mediterranean Area. Meteorologische Zeitschrift, 21, 61-77.

https://doi.org/10.1127/0941-2948/2012/0271

[6] Dmitriev, A.V. and Suvorova, A.V. (2015) Large-Scale Jets in the Magnetosheath and Plasma Penetration across the Magnetopause: Themis Observations. Journal of Geophysical Research Space Physics, 120, 4423-4437.

https://doi.org/10.1002/2014JA020953

[7] Volkova, E.V. and Uspensky, A.B. (2016) Detection and Assessment of Cloud cover and Precipitation Parameters Using Data of Scanning Radiometers of Polar-Orbiting and Geostationary Meteorological Satellites. Izvestiya Atmospheric \& Oceanic Physics, 52, 1097-1109. https://doi.org/10.1134/S0001433816090280

[8] Behrendt, A., Pal, S., Aoshima, F., Bender, M., Blyth, A., Corsmeier, U., et al. (2015) Observation of Convection Initiation Processes with a Suite of State-of-the-Art Research Instruments during Cops Iop 8b. Quarterly Journal of the Royal Meteorological Society, 137, 81-100. https://doi.org/10.1002/qj.758

[9] Loeb, N.G. and Wielicki, B.A. (2015) Satellites and Satellite Remote Sensing Earth's Radiation Budget. Encyclopedia of Atmospheric Sciences, 85, 67-76. https://doi.org/10.1016/B978-0-12-382225-3.00349-2

[10] Brunke, M.A., Stegall, S.T. and Zeng, X. (2015) A Climatology of Tropospheric Humidity Inversions in Five Reanalyses. Atmospheric Research, 153, 165-187. https://doi.org/10.1016/j.atmosres.2014.08.005

[11] Jiang, X., Waliser, D.E., Xavier, P.K., Petch, J., Klingaman, N.P., Woolnough, S.J., et al. (2015) Vertical Structure and Physical Processes of the Madden-Julian Oscillation: Exploring Key Model Physics in Climate Simulations. Journal of Geophysical Research Atmospheres, 120, 4718-4748. https://doi.org/10.1002/2014JD022375

[12] Conover, J.H. (2015) The Interpretation of Cloud Pictures from the Tiros Meteorological Satellites. Journal of the SMPTE, 71, 21-25. https://doi.org/10.5594/J16909

[13] Peng, Y., Mi, K., Qing, F., Liu, X., Liu, L. and Chen, Q. (2015) Ecological Risk Assessment for Key Industrial Development Zones in the Areas Surrounding the Bo Sea in China. Human \& Ecological Risk Assessment an International Journal, 22, 475-488. https://doi.org/10.1080/10807039.2015.1080592

[14] Dee, S., Emile-Geay, J., Evans, M.N., Allam, A., Steig, E.J. and Thompson, D.M. (2015) Prysm: An Open-Source Framework for Proxy System Modeling, with Applications to Oxygen-Isotope Systems. Journal of Advances in Modeling Earth Systems, 7, 1220-1247. https://doi.org/10.1002/2015MS000447

[15] Yu, X.X., Yin, H., Li, H.X., Zhang, W., Zhao, H., Li, C., et al. (2017) Piezo-Phototronic Effect Modulated Self-Powered Uv/Visible/Near-Infrared Photodetectors Based on Cds: p3ht Microwires. Nano Energy, 34, 155-163. 
https://doi.org/10.1016/j.nanoen.2017.02.033

[16] Delory, G.T., Luhmann, J.G., Brain, D., Lillis, R.J., Mitchell, D.L., Mewaldt, R.A., et al. (2016) Energetic Particles Detected by the Electron Reflectometer Instrument on the Mars Global Surveyor, 1999-2006. Space Weather-The International Journal of Research \& Applications, 10, 1-23.

[17] Da, C. (2014) An Objective Regional Cloud Mask Algorithm for Goes Infrared Imager with Regime-Dependent Thresholds for Direct Radiance Assimilation. Dissertations \& Theses-Gradworks, 119, 6666-6680.

[18] Song, G., Subrahmanyam, M.V., Guo, B. and Panda, J. (2015) A Case Study of Polar Low Detection using ers-2 Wave Mode Image. Open Oceanography Journal, 8, 28 32. https://doi.org/10.2174/1874252101408010028

[19] Suliman, A. and Zhang, Y. (2018) Disparity-Based Generation of Line-of-Sight DSM for Image-Elevation Co-Registration to Support Building Detection in OffNadir VHR Satellite Images. Journal of Geographic Information System, 10, 25-56. https://doi.org/10.4236/jgis.2018.101002

[20] Hermi, S., Abdalla Elsheikh, R., Aziz, M. and Bouaziz, S. (2017) Structural Interpretation of Lineaments Uses Satellite Images Processing: A Case Study in NorthEastern Tunisia. Journal of Geographic Information System, 9, 440-455.

https://doi.org/10.4236/jgis.2017.94027

[21] Saleem, M. and Ahmed, S. (2016) Missing Data Imputations for Upper Air Temperature at 24 Standard Pressure Levels over Pakistan Collected from Aqua Satellite. Journal of Data Analysis and Information Processing, 4, 132-146. https://doi.org/10.4236/jdaip.2016.43012

[22] Zhao, X., Lin, M., Lin, W., Xie, L. and Fan, C. (2017) Research on Exception of Meteorological Satellite Ground System Application Based on Resource Bottleneck. Atmospheric and Climate Sciences, 7, 367-373. https://doi.org/10.4236/acs.2017.73027

[23] Laudares, S., Laudares, J. and Libório, M. (2016) Geographic Information Systems Used as a Practical Way of Teaching Mathematics. Journal of Geographic Information System, 8, 608-617. https://doi.org/10.4236/jgis.2016.85050 